\title{
Diagnosis and management of pancreatic cystic neoplasms
}

\author{
Mathew James Keegan, ${ }^{\oplus 1,2}$ Bharat Paranandi ${ }^{3}$
}

${ }^{1}$ Gastroenterology, Northern Beaches Hospital, Sydney, New South Wales, Australia

${ }^{2}$ Department of

Gastroenterology, Peninsula Gastroenterology, Sydney, New

South Wales, Australia

${ }^{3}$ Gastroenterology, Leeds

Teaching Hospitals NHS Trust, Leeds, UK

\section{Correspondence to}

Dr Bharat Paranandi, Gastroenterology, Leeds Teaching Hospitals NHS Trust, Leeds LS1 3EX, UK; b.paranandi@nhs.net

Received 18 October 2018 Revised 18 January 2019 Accepted 19 January 2019 Published Online First 1 March 2019

\section{SLinked}

- http://dx.doi.org/10.1136/ flgastro-2019-101192

\section{Check for updates}

(C) Author(s) (or their employer(s)) 2019. No commercial re-use. See rights and permissions. Published by BMJ.

To cite: Keegan MJ,

Paranandi B. Frontline

Gastroenterology

2019;10:300-308.

\begin{abstract}
This review outlines the current classification of pancreatic cystic lesions, with a particular emphasis on pancreatic cystic neoplasms (PCNs). It will describe the diagnostic approach to PCNs, with reference to clinicopathological features, cross-sectional radiology and endoscopic ultrasound. This review will conclude with an evidence-based discussion of the management of PCNs focused on recent clinical guidelines.
\end{abstract}

Pancreatic cystic lesions (PCLs) are frequent incidental findings with modern cross-sectional imaging. The prevalence of cysts $\geq 2 \mathrm{~mm}$ in imaging series is $4 \%-45 \%$, increasing with age. ${ }^{1-3}$ The WHO 2010 classification of pancreatic tumours assigns PCL into four categories based on the presence or absence of an epithelialised cyst lining and neoplastic potential. ${ }^{4}$ Epithelialised neoplastic cysts include the pancreatic cystic neoplasms (PCNs), cystic degeneration of solid tumours and rarer tumours. ${ }^{4}$

\section{CLASSIFICATION OF PANCREATIC CYSTIC NEOPLASMS}

Even in high volume centres, pancreatic surgery carries significant morbidity and mortality, and classification is key to establishing appropriate management or surveillance. Pseudocysts are an important benign differential, while malignant mimics include cystic neuroendocrine tumours and cystic degeneration of solid tumours.

In our experience (tertiary pancreaticobiliary cancer centre), we advise that most incidentally discovered or indeterminate aetiology PCLs are referred for central review and management discussion. The majority of PCLs reviewed at multidisciplinary team meetings are intraductal papillary mucinous neoplasms (IPMN), pseudocysts or mucinous cystic neoplasms (MCN). Serous cystic neoplasms (SCN) are less commonly referred due to definitive diagnosis with good cross sectional imaging. Other PCLs including solid pseudopapillary neoplasms (SPN) are far less common.

\section{Serous}

Serous cystic neoplasm

Historically termed 'microcystic adenomas', these are slowly growing lesions with fibrous structures forming a honeycomb appearance within a well-defined capsule. A central, stellate scar is seen in $30 \%$ on CT. About $10 \%-20 \%$ of SCN are oligocystic or macrocystic and can be confused with mucinous cysts. Lobulated contrast enhancement in the cyst capsule may be a clue to differentiating an oligocystic SCN from $\mathrm{MCN}^{56}$

Peak incidence is in the seventh decade of life (75\% females). These are most often discovered incidentally, typically in the pancreatic body and tail. Symptoms related to mass effect are occasionally present-anorexia, weight loss, abdominal pain, palpable mass or jaundice. ${ }^{78}$ Lesions are typically unifocal, though multiple cysts may be seen in patients with Von Hippel Lindau Syndrome, 90\% of whom have SCN. ${ }^{9}$ The classic endoscopic ultrasound (EUS) appearance is of a multicystic lesion with small anechoic cysts and intervening hypoechoic areas. Fluid amylase levels and fluid carcinoembryonic antigen (CEA) are not elevated and cyst fluid is not viscous. ${ }^{10}$ Cytology is often acellular, though may reveal glycogen-rich cuboidal epithelial cells. Fine needle aspirate (FNA) is often bloody. ${ }^{11}$

The differentiation between serous cystic adenoma (SCA) and serous cystic carcinoma (SCC) is dependent on the demonstration of malignant potential. ${ }^{4}$ There have been very few reported cases of malignant transformation and surveillance is typically not recommended. ${ }^{12}{ }^{13}$ SCNs are not routinely resected. ${ }^{14}$

Controversy exists around the role for surveillance or surgery when SCAs are 
diagnosed in younger patients. Indications for resection include suspicion of SCC, significant symptoms and size $>4 \mathrm{~cm}$ (though size is known to poorly correlate with likelihood of malignancy). Prognosis postresection is excellent, including reports of long-term postoperative survival. ${ }^{15}$

\section{Solid pseudopapillary neoplasms}

SPN are typically large lesions within a fibrous capsule comprised of mixed solid and cystic components. Microscopically, monomorphic epithelial cells form pseudopapillae and haemorrhagic necrosis creates cystic foci. Malignancy is seen in 10\%-20\%. Lymph node involvement and distant metastases have been reported in cases without features of local invasion. ${ }^{16-18}$ Molecularly, SPN differs from pancreatic ductal adenocarcinoma (PDAC) by activation of the beta-catenin pathway in most tumours and frequent expression of progesterone receptors. ${ }^{19}$ Historically SPN present due to mass effect, with symptoms similar to SCNs. Asymptomatic presentations are increasing. More than $80 \%$ of SPNs present in females, typically in the fourth decade. Paediatric presentations comprise up to $25 \%$ of SPNs. ${ }^{16-18}$

Cross-sectional imaging reveals a well-demarcated fibrous capsule surrounding a heterogeneous solid-cystic mass. EUS appearance is similar, with a well-defined hypoechoic mass without MPD communication or dilatation. The extent of the solid or cystic components may vary widely. EUS FNA is typically bloody, with low viscosity and low amylase/CEA levels in keeping with a non-mucinous cyst. Cytological features are of pseudopapillary structures and fibrovascular tissue cores with variable eosinophilia.

Surgical resection is favoured due to malignant potential, early median age of diagnosis and symptomatic mass effect. ${ }^{14}$ Post-resection 5 year survival rates are as high as $95 \%$; however, ongoing surveillance imaging is recommended as the risk of recurrence $(10 \%)$ extends beyond 5 years. ${ }^{820}$

\section{Mucinous}

Mucinous cystic neoplasms

MCNs are solitary lesions, occurring almost universally in the body or tail of pancreas (>97\%) of females $(>98 \%)$ in their fifth and sixth decade of life. ${ }^{21} 22$ Histologically, these uniloculated or multiloculated cysts are lined by mucin producing duct type epithelium within a fibrous capsule of ovarian type stroma (spindle cells and epithelioid cells). Uniloculated MCNs have been mistaken for pseudocysts and multiloculated lesions may be difficult to differentiate from SCN prior to cyst fluid analysis. $^{823}$

MCNs are, predominately, an asymptomatic finding. The features related to age, gender, solitary nature and location within the pancreas may give strong clues to the diagnosis. Most do not exhibit MPD communication, central scarring is absent and up to $15 \%$ contain calcifications of the peripheral capsule. ${ }^{24}$

MPD communication may be more readily excluded at EUS to differentiate these lesions from branch duct-intraductal papillary mucinous neoplasms (BD-IPMN), though prior endoscopic retrograde pancreatography demonstrating MPD communication has been reported in up to $15 \%$ of histologically confirmed $\mathrm{MCN} .{ }^{25} \mathrm{MCN}$ is often strongly suspected on clinicoradiological and demographic criteria. EUS is superior to cross-sectional imaging in detecting mural nodules, which along with size $>4 \mathrm{~cm}$, is a predictor of malignant behaviour. Fluid analysis typically reveal viscous aspirate with high CEA consistent with a mucinous lesion. Variability in MPD communication likely explains the variation in amylase levels, though this is typically low (a potential point of differentiation with IPMN). Cytology may demonstrate mucinous columnar epithelial cells within background mucin. ${ }^{82326}$

Cancer is reported in 4\%-30\% of resected MCNs. ${ }^{23} 24$ In MCNs without high grade dysplasia (HGD), resection is considered curative. For those with established malignancy at the time of diagnosis, prognosis is much poorer and postresection surveillance is in line with recommendations given for PDAC. ${ }^{2326}$

\section{Intraductal papillary mucinous neoplasms}

IPMNs are mucin producing tumours, which in contrast to other PCNs, characteristically arise in (main duct IPMN; MD-IPMN) or communicate with (BD-IPMN) the MPD. A mucinous cyst in communication with a focally or diffusely ectatic MPD are said to be of mixed type (MT-IPMN) (figure 1). Another point of distinction from other PCNs is that BD-IPMNs are multifocal in $10 \%-40 \%$ of cases. ${ }^{28} 29$ The invariably dysplastic mucosa of these lesions is analogous in appearance to tubulovillous adenoma of the colon, with columnar epithelium and goblet cells covering prominent papillae (producing copious mucin). There are four epithelial subtypes; gastric (seen in BD-IPMN), intestinal, pancreaticobiliary and oncolytic (latter three seen in MD-IPMN). The Gastric subtype confers the best prognosis and pancreaticobiliary subtype the least favourable. ${ }^{30}$ It is not routinely possible to determine the epithelial subtype prior to surgery.

The typical IPMN presents in the sixth or seventh decade of life as an asymptomatic lesion in the head or neck of pancreas $(70 \%)$. If symptomatic, the classical presentation is of recurrent acute pancreatitis in a patient with a dilated MPD without features of, or risk factors for, chronic pancreatitis. ${ }^{2328} 29$ IPMNs are seen on up to $20 \%$ of MRI scans visualising the pancreas. ${ }^{31}$ MD-IPMN can be suspected in patients with focal or diffuse ectasia of the MPD (>5 mm). BD-IPMN are cystic dilatations of pancreatic duct side branches, though MPD communication is not universally appreciable on imaging. If an IPMN is suspected, it can be better characterised by 

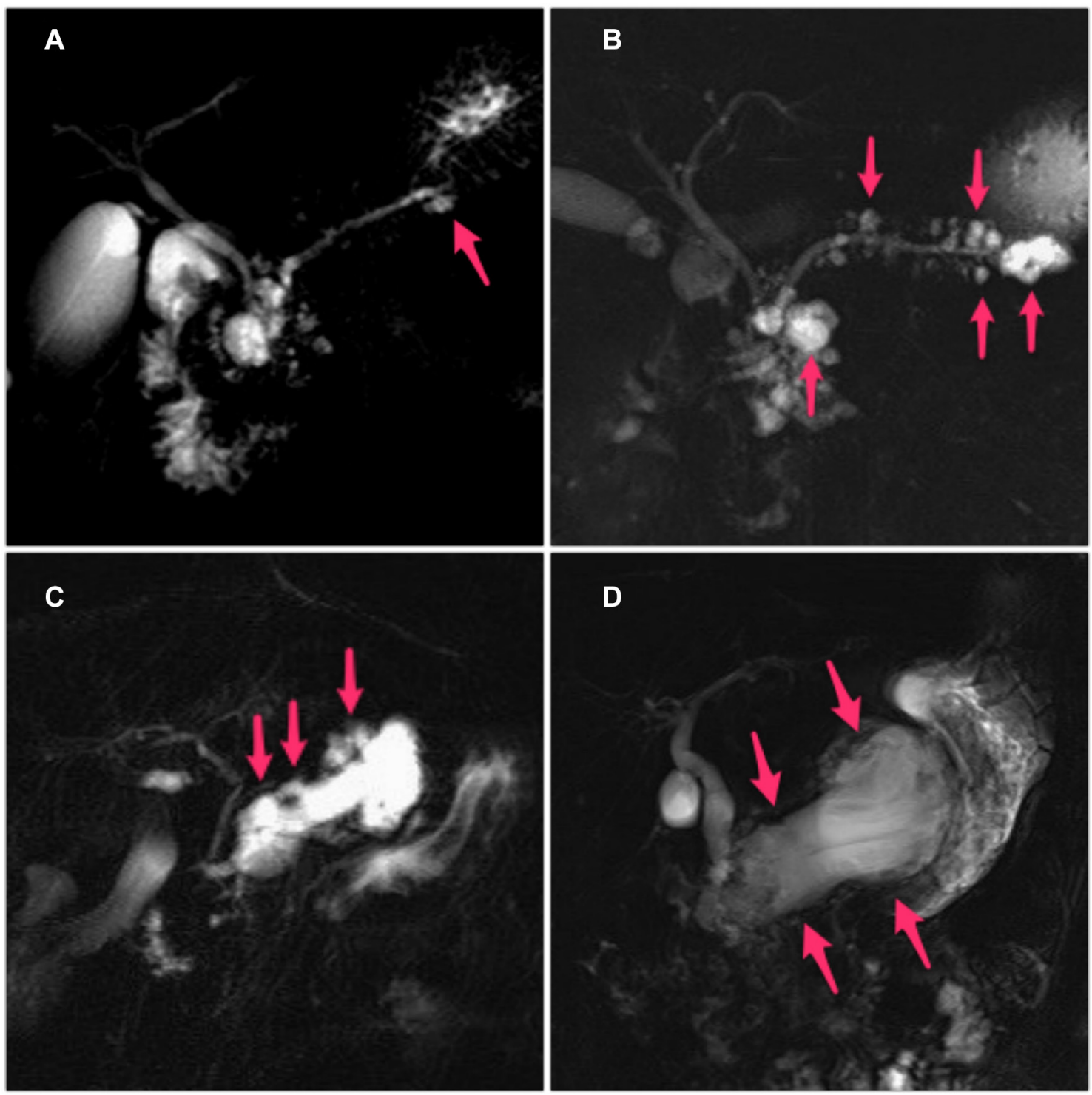

Figure 1 Magnetic resonance cholangio-pancreatogram characterisation of intraductal pancreatic mucinous neoplasm: (A) branch duct type, (B) multifocal branch duct type, (C) mixed type, (D) main duct type.

either pancreas protocol CT or gadolinium enhanced MRI pancreas with MRCP. 2330

All IPMN harbour malignant potential. The goal of diagnostic work-up is to determine evidence for MPD involvement or features of invasion as these patients should be considered for resection if they are fit surgical candidates. ${ }^{1430}$

EUS is superior to cross-sectional imaging for confirming MPD communication and for the detection of mural nodules as well as for exclusion of alternative diagnoses (eg, pseudocyst or chronic pancreatitis). ${ }^{32}$ EUS FNA with fluid analysis allows determination of mucinous component as well as cytological analysis which is moderately sensitive, but highly specific for malignancy. ${ }^{33}$ Further details on assessment, management and surveillance of IPMN follows below.

\section{APPROACH TO DIAGNOSTIC EVALUATION OF PANCREATIC CYSTIC NEOPLASMS}

1. Determine the type of cyst.

2. Scrutinise for current malignant behaviour.

3. Establish the risk of future malignant transformation.

The clinical context and patient's fitness for surgery must first be established. Demographics may give clues to the likely aetiology. While $70 \%$ of patients will be asymptomatic, symptoms such as pain, weight loss or jaundice should prompt emergent exclusion of invasive disease. Pancreatitis in the context of excess alcohol or gall stones should prompt exclusion of an inflammatory aetiology. Enquiries should also be made into a possible family history of pancreatic adenocarcinoma or genetic syndromes. ${ }^{3} 3034$

This review has drawn largely from the The European Study Group on Cystic Tumours of the Pancreas 
Table 1 Risk stratifying characteristics of IPMN used in the Revised Fukuoka Guidelines ${ }^{30}$

\begin{tabular}{|c|c|}
\hline Characteristics & Action if present \\
\hline \multicolumn{2}{|l|}{ Cross-sectional imaging } \\
\hline $\begin{array}{l}\text { High-risk stigmata } \\
\text { Obstructive jaundice associated with head } \\
\text { of pancreas cyst } \\
\text { Enhancing mural nodule } \geq 5 \mathrm{~mm} \\
\text { Main pancreatic duct } \geq 10 \mathrm{~mm}\end{array}$ & $\begin{array}{l}\text { Consider surgery in fit } \\
\text { patients }\end{array}$ \\
\hline $\begin{array}{l}\text { Worrisome features } \\
\text { Clinical-pancreatitis } \\
\text { Radiological } \\
\text { Cyst } \geq 3 \mathrm{~cm} \\
\text { Enhancing mural nodule }<5 \mathrm{~mm} \\
\text { Thickened/enhancing cyst walls } \\
\text { Main pancreatic duct size } 5-9 \mathrm{~mm} \\
\text { Abrupt change in calibre of pancreatic duct with } \\
\text { distal pancreatic atrophy } \\
\text { Lymphadenopathy } \\
\text { Elevated serum CA19-9 } \\
\text { Cyst growth rate } \geq 5 \mathrm{~mm} / 2 \text { years }\end{array}$ & $\begin{array}{l}\text { Perform endoscopic } \\
\text { ultrasound evaluation }\end{array}$ \\
\hline $\begin{array}{l}\text { EUS features } \\
\text { Definite mural nodule } \geq 5 \mathrm{~mm} \\
\text { Main duct features suspicious for involvement } \\
\text { Cytology suspicious or definite for } \\
\text { malignancy }\end{array}$ & $\begin{array}{l}\text { Consider surgery in fit } \\
\text { patients }\end{array}$ \\
\hline
\end{tabular}

IPMN, intraductal papillary mucinous neoplasms.

2018 European evidence-based guidelines on pancreatic cystic neoplasms (ESGCTP guidelines) and the 2017 revised Fukuoka guidelines, both offer current, detailed guidance to clinical practice. ${ }^{30}$ The 2018 American College of Gastroenterology guidelines are broadly in agreement with these. ${ }^{34}$ The 2015 American Gastroenterology Association guidelines offer less guidance on the management of complex patients, and some of their recommendations have been controversial, such as discontinuing surveillance after 5 years. ${ }^{35}$

\section{Cross-sectional imaging}

Invasive foci in asymptomatic patients with cystic lesions $<5 \mathrm{~mm}$ in size are rare. Cysts $>5 \mathrm{~mm}$ should be first evaluated by pancreas protocol CT or MRI. While CT is readily available, MRI gives enhanced resolution of nodules and septae, better delineates duct communication and for these reasons is favoured by recent guidelines ${ }^{3034}$ (figure 1).

The 2017 revised Fukuoka guidelines outline 'high risk stigmata' and 'worrisome features' (see table 1). They recommend surgical referral for 'high risk stigmata' and EUS assessment for 'worrisome features'. IPMN $2-3 \mathrm{~cm}$ in size without 'worrisome features' are advised to have EUS in 3-6 months and smaller cysts to have CT or MRI based surveillance with intervals based on size ${ }^{30}$ (table 1). The ESGCTP guidelines recommend referral for surgical review for all patients with 'absolute indications' and referral based on the consideration of comorbidities and number of risk factors for those with 'relative indications' (table 2). ${ }^{3}$
Table 2 Indications for surgery in IPMN from the 2018 ESGCTP Guidelines $^{3}$

\begin{tabular}{|c|c|}
\hline Characteristics & $\begin{array}{l}\text { Conditions for surgical } \\
\text { referral }\end{array}$ \\
\hline \multicolumn{2}{|l|}{ Absolute indications for surgery } \\
\hline $\begin{array}{l}\text { Positive cytology for malignancy/high } \\
\text { grade dysplasia } \\
\text { Solid mass } \\
\text { Tumour related jaundice } \\
\text { Enhancing mural nodule } \geq 5 \mathrm{~mm} \\
\text { Main pancreatic duct } \geq 10 \mathrm{~mm}\end{array}$ & - All fit patients \\
\hline $\begin{array}{l}\text { Relative indications for surgery } \\
\text { Cyst growth rate } \geq 5 \mathrm{~mm} / \text { year } \\
\text { Elevated serum CA19.9 (>37 U/mL) } \\
\text { Cyain pancreatic duct } 5-9.9 \mathrm{~mm} \\
\text { Cyst diameter } \geq 40 \mathrm{~mm} \\
\text { New onset diabetes mellitus } \\
\text { Acute pancreatitis (secondary to IPMN) } \\
\text { Enhancing mural nodule ( }<5 \mathrm{~mm})\end{array}$ & $\begin{array}{l}\text { No significant } \\
\text { comorbidities and } \geq 1 \\
\text { relative indication } \\
\text { Significant } \\
\text { comorbidities and } \geq 2 \\
\text { relative indications }\end{array}$ \\
\hline
\end{tabular}

ESGCTP, The European Study Group on Cystic Tumours of the Pancreas; IPMN, intraductal papillary mucinous neoplasms.

Positron emission tomography (PET) has utility in detecting distant metastases in the setting of confirmed PDAC preventing unnecessary surgery. ${ }^{36}$ However, the differentiation of benign from malignant PCN using PET produces variable results. ${ }^{37-40}$ Using PET for identifying metastatic lesions in patients with high risk features (being considered for surgery) may prove more fruitful.

\section{Endoscopic ultrasound}

EUS should be reserved for cases where it is expected to alter management. The high resolution of soft tissue may allow visualisation of septae, nodules, internal debris/mucin and duct communication ${ }^{41}$ (figure 2). In a retrospective review of 145 cases, EUS demonstrated multifocal IPMN resulting in a change to the surgical field for a significant number of patients compared with both CT (47\% vs $13 \%)$ and MRI (33\% vs 4\%). Other trials have not demonstrated an advantage of diagnostic EUS over CT or MRI in the preoperative diagnosis of $\mathrm{PCN}^{42}{ }^{43}$ Detection of high risk features at EUS should prompt surgical referral. The 2018 ESGCTP guidelines recommend performing EUS at any point at which a $\mathrm{PCN}$ develops clinical or radiology concerns. ${ }^{3}$

Contrast-harmonic enhanced (CHE) EUS can allow demonstration of blood flow to suspected mural nodules, delineating these from mucin and internal debris, as recommended by the 2018 ESGCTP. $^{3}$

\section{Cyst fluid analysis}

An advantage of EUS is the ability to sample cyst fluid. Cyst FNA is invasive and should be performed when expected to alter management, such as IPMN with 'worrisome features' or in undifferentiated PCNs. Relative contraindications include the need to traverse $>10$ $\mathrm{mm}$ of pancreatic parenchyma, high-risk bleeding disorders and use of dual antiplatelet therapy. ${ }^{3}$ 

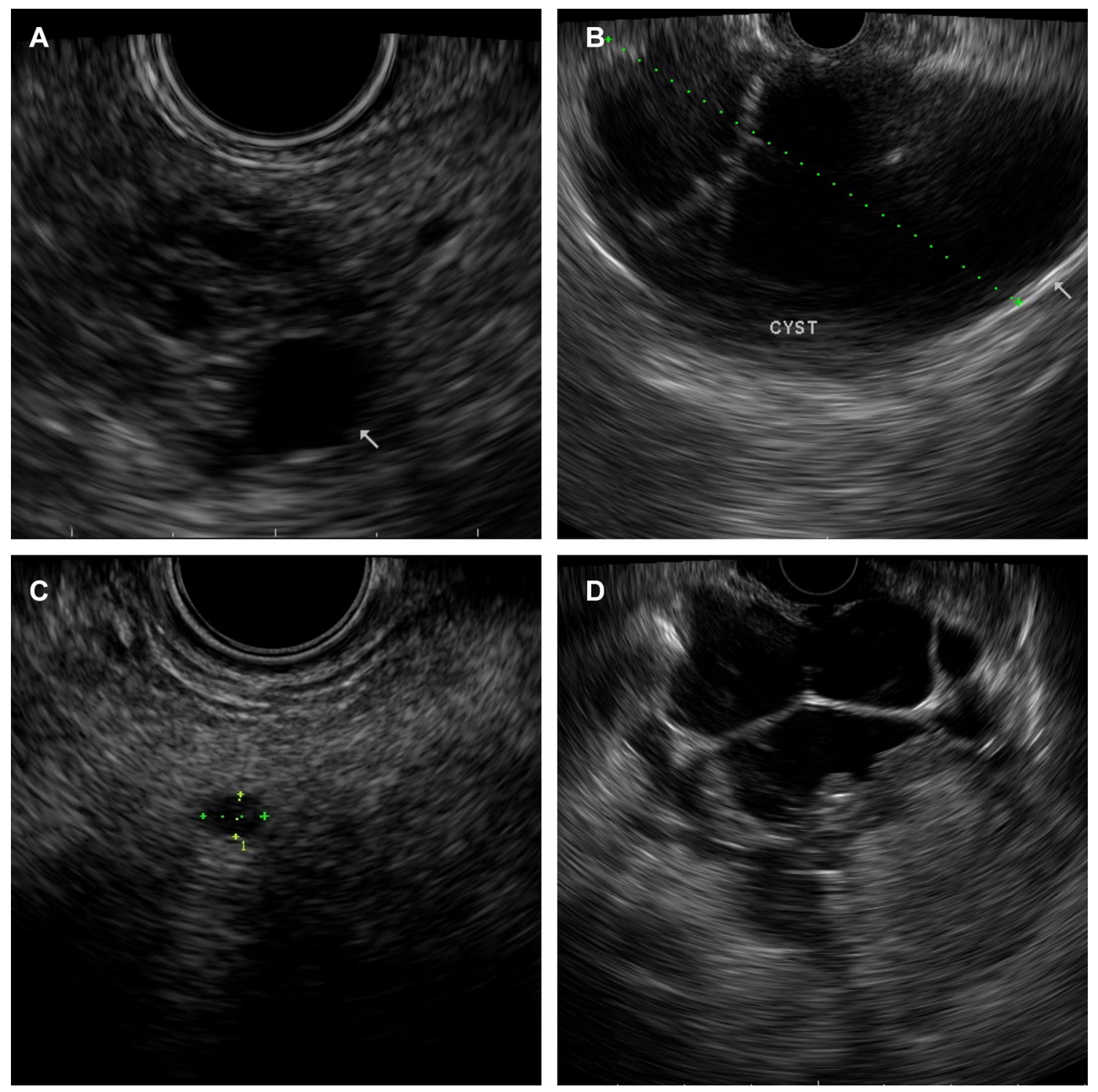

Figure 2 EUS imaging of pancreatic cystic neoplasms: (A) serous cystic neoplasm, (B) mucinous cystic neoplasm, (C) branch duct type intraductal papillary mucinous neoplasm, (D) malignant changes within an intraductal papillary mucinous neoplasm.

A CEA level $>192 \mathrm{ng} / \mathrm{mL}$ has a sensitivity of $73 \%$ and specificity of $84 \%$ for the diagnosis of a mucinous lesion. ${ }^{44}$ Below $5 \mathrm{ng} / \mathrm{mL}$ and above $800 \mathrm{ng} / \mathrm{mL}$, the negative and positive predictive values, respectively for mucinous aetiology are both $94 \% .{ }^{45}$ Mucin may be seen on cytological analysis or suggested by a positive 'string test'. ${ }^{46}$ Elevated amylase/lipase reflects duct communication, as in IPMN and pseudocysts. Variable amylase/ lipase levels seen in MCNs make them an imperfect tool for differentiating mucinous cyst types. The 2018 ESGCTP guidelines advise that the combination of cytology, CEA and lipase provides the highest diagnostic accuracy in differentiating mucinous from non-mucinous PCN. ${ }^{3}$

Low cellularity hampers cytological detection of malignancy and differentiation of PCN type. The sensitivity for detecting HGD and carcinoma in IPMN is only $50 \%$, though as high as $72 \%$ if limited to use in cysts with worrisome features. ${ }^{30}$ Its strength is in its specificity-suspicious or malignant cytology should prompt surgical referral.

Despite these limitations, EUS FNA reduces inappropriate surgery for asymptomatic serous lesions and inappropriate surveillance of high-risk lesions under $3 \mathrm{~cm}$ in size. ${ }^{47}$ Doing so alters management in $36 \%$ of patients evaluated by CT and 54\% of those evaluated by MRI. ${ }^{48}$

\section{Other adjuncts to endoscopic evaluation of pancreatic cystic neoplasms}

A pouting, 'fish-mouth', major ampulla, with appreciably mucoid secretions is indicative of main duct involvement by IPMN. This is best seen with a side-viewing duodenoscope. $^{30}$

Pancreatoscopy allows visualisation of the papillary projections of MD-IPMN and MT-IPMN facilitating directed biopsy, distinction from other causes of dilated 
MPD and determination of the extent of MPD involvement preoperatively. ${ }^{49}$

The endoscopic evaluation of PCNs is not dependent on any one diagnostic feature, but rather relies on the synthesis of information from the EUS imaging, cytology, cyst fluid analysis and any diagnostic adjuncts. This was eloquently demonstrated by Oppong and colleagues who showed that a combination EUS diagnosis (endosonographic impression, cytology and CEA level) had a sensitivity (91\%), specificity (75\%) and accuracy $(85.7 \%)$ higher than any test component in isolation ( $\mathrm{p}<0.05$, for all comparisons). ${ }^{50}$

\section{Serum}

CA 19-9 has been evaluated in the preoperative setting. Sensitivities and specificities ranging from $56 \%$ to $75 \%$ and $74 \%$ to $96 \%$ have been reported for the presence of adenocarcinoma at resection. ${ }^{51-53}$ The ESGCTP includes CA19.9 in the routine surveillance algorithm for IPMN. ${ }^{3}$

\section{APPROACH TO MANAGEMENT}

A major hindrance in the management of PCN relates to establishing a histological diagnosis in cysts which are not resected. Cysts with an uncertain diagnosis should be managed as per mucinous cysts. ${ }^{3}$

\section{Surgical resection}

While universal resection of MCN had been recommended ${ }^{26}$ there is a paucity of evidence for this approach. ${ }^{35}$ ESGCTP guidelines recommend resection of $\mathrm{MCN} \geq 40 \mathrm{~mm}$, if symptomatic or if harbouring risk factors such as mural nodules. ${ }^{3}$ These lesions are typically found in the distal pancreas of younger patients and may be amendable to less morbid distal pancreatectomy.

Most guidelines consider MD-IPMN and BD-IPMN separately, based on mean rates of HGD or invasive disease, of 62\% in MD-IPMN. The 2017 revised Fukuoka guidelines quote 5-year survival rates for resected MD-IPMN far superior to PDAC (31\%-54\%) and recommend resection of MD-IPMN in surgically fit patients with MPD $>10 \mathrm{~mm}$, jaundice or mural nodules. ${ }^{30}$ The 2018 ESGCTP guidelines go further. While similarly including MPD>10 mm as an 'absolute indication' for surgery, they consider MPD of 5-9.9 $\mathrm{mm}$ as a 'relative indication', citing published rates of HGD or cancer of 37\%-91\% (vs 56\%-89\% in MPD $>10 \mathrm{~mm}){ }^{3}$ In diffusely dilated MPD, efforts should be made to establish the extent of duct involvement. This can determine the suitability for segmental resection and ensure these criteria are not mistakenly applied to other causes of MPD dilatation (eg, chronic pancreatitis). This could be established preoperatively with intraductal ultrasound or pancreatoscopy or by intraoperative frozen section. ${ }^{49} 5455$

A considerable number of BD-IPMN harbour HGD or invasive carcinoma $(14 \%-48 \%)$ at diagnosis and should be resected. ${ }^{56-59}$ The remainder have a low annualised rate of malignant progression (1\%-7\%) lending themselves to surveillance. ${ }^{60-62}$ The 2017 Fukuoka guidelines indications for surgery from cross-sectional imaging are described in table 1. EUS criteria prompting surgical referral are then mural nodule $\geq 5 \mathrm{~mm}, \mathrm{MPD}$ involvement and cytology suspicious or positive for malignancy. ${ }^{30}$ The 2018 ESGCTP list similar 'absolute indications' for surgery (table 2). Their relative indications (table 2) include biochemical, cyst and clinical features to be considered on the balance of patient comorbidities. ${ }^{3}$ In both guidelines, patients not meeting these criteria enter surveillance as described below.

Multifocal disease, present in 14\%-41\% of BD-IPMN, should be considered when planning the type of resection. ${ }^{63-65}$ The physiological function of residual pancreas must be taken into account. The ESGCTP recommend resection only of those cysts exhibiting indications for resection, with per protocol surveillance of unresected cysts. $^{3}$

\section{Surveillance}

After consideration of the 'high-risk stigmata' and 'worrisome criteria', the 2017 revised Fukuoka guidelines stratifies patients by cyst size. EUS is indicated both in cysts $>3 \mathrm{~cm}$ (alternating every 3-6 months with MRI) and in cysts $2-3 \mathrm{~cm}$ (EUS within 3-6 months of detection and then annual surveillance alternating EUS and MRI). Cysts of $1-2 \mathrm{~cm}$ are monitored by CT/MR 6 monthly for 1 year, annually for 2 years, then biannually if no changes detected, while those $<1 \mathrm{~cm}$ are reassessed at 6 months before biannual surveillance with CT/MR. Any concerning evolution not meeting the criteria for surgery should prompt shortening of surveillance interval (3-9 months) (figure 3). ${ }^{30}$

The 2018 ESGCTP guidelines recommend clinical and radiological (MRI and/or EUS) follow-up 6-monthly in the first year, then annually, for both IPMN and MCN. For IPMN they advise serum CA19.9 at each of these time points. ${ }^{3}$

The 2017 revised Fukuoka guidelines highlights the not infrequent development of both new IPMN and concurrent PDAC lesions, regardless of the presence of HGD at resection, with reports of this occurring beyond 10 years postresection. ${ }^{30}$ They reference a 10 -year IPMN recurrence rate of $62 \%$ with invasive IPMN in $38 \% .6667$ They recommend at least twice yearly surveillance with cross-sectional imaging in high risk patients (family history of PDAC, HGD at resection margin and non-intestinal IPMN subtype) and 6-12 monthly surveillance in others for as long as they remain candidates for surgical resection. ${ }^{30}$ The 2018 ESGCTP guidelines recommend surveillance as long as the patient remains fit for surgery. Any patients with foci of carcinoma are followed as per PDAC, patients with HGD or MPD involvement should be followed 6-monthly for 2 years and then annually, while all others should be followed as per unresected cysts. ${ }^{3}$ 
Are any of the following "high-risk stigmata" of malignancy present?

i) obstructive jaundice in a patient with cystic lesion of the head of the pancreas, ii) enhancing mural nodule $\geq 5 \mathrm{~mm}$,

iii) main pancreatic duct $\geq 10 \mathrm{~mm}$

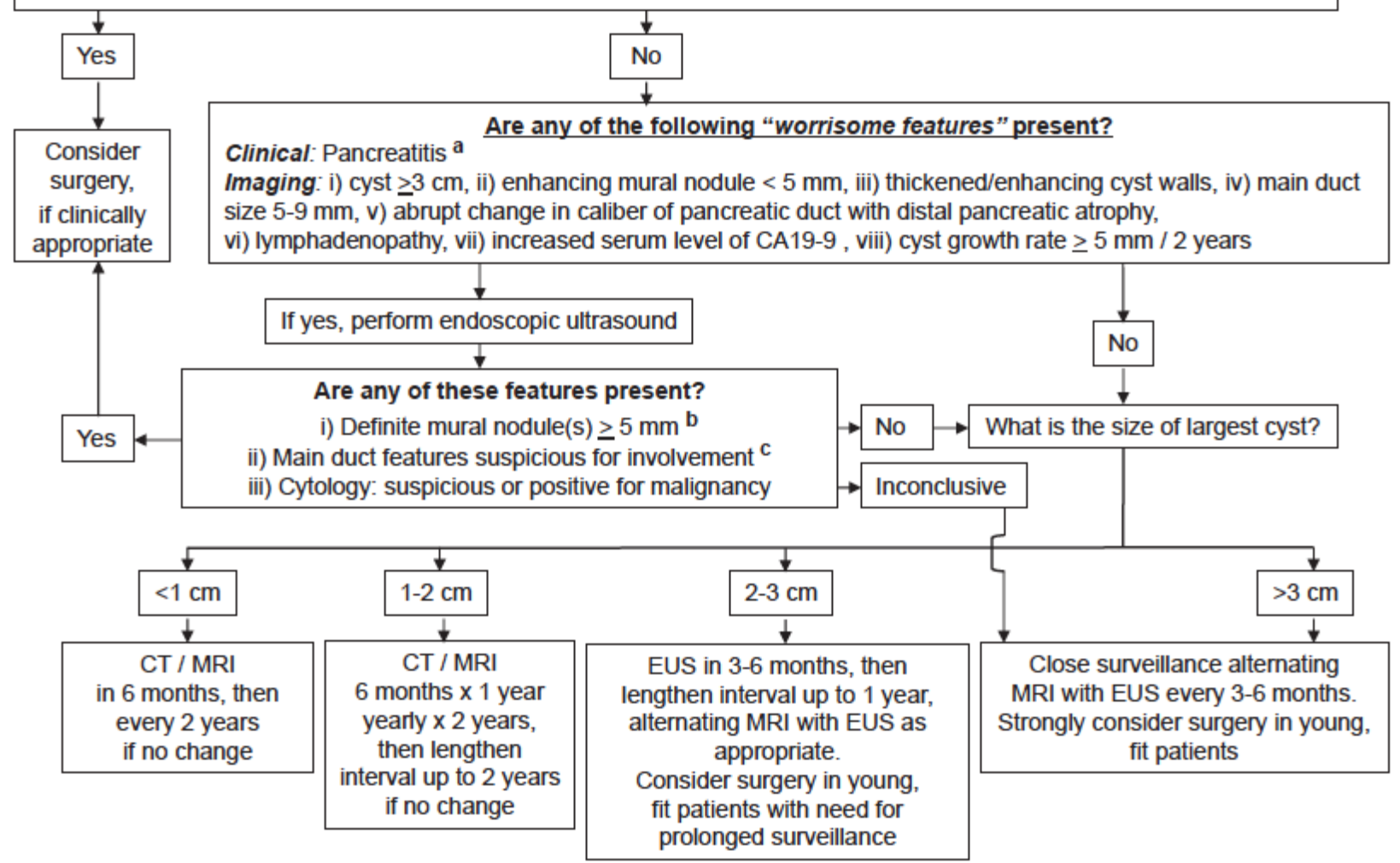

Figure 3 Algorithm for the management of suspected BD-IPMN. ${ }^{5}$ Reprinted with permission from Tanaka M. ${ }^{30}$ Copyright 2017, with permission from Elsevier. BD-IPMN, branch duct-intraductal papillary mucinous neoplasms; EUS, endoscopic ultrasound.

\section{Can we stop surveying?}

The 2015 AGA guidelines aside, all other guidelines recommend continuation of surveillance of all unresected IPMN for as long as the patient remains fit for surgery. ${ }^{3} 3034$

\section{Future directions}

A recent study by Krishna and colleagues investigating intracystic needle confocal laser endomicroscopy demonstrated sensitivities, specificities and diagnostic accuracies $\geq 94 \%$ for mucinous cysts and $\geq 98 \%$ for SCAs. Intraobserver reliability and inter-observer agreement were near perfect for mucinous cysts and SCAs (kappa values $>0.80) .{ }^{68}$ Recently published guidelines advised awaiting large prospective studies before this technique is recommended outside research settings. ${ }^{3}$ Microbiopsy forceps deployed via a 19 gauge FNA needle may improve sample cellularity, though robust data does not yet exist. ${ }^{31}$

There has been much interest in the development of DNA and molecular markers to improve cyst fluid analysis. K-ras proto-oncogene mutations strongly correlate with mucinous cysts, and their concurrence with aneuploidy or allelic loss is $96 \%$ specific for malignancy. ${ }^{69} 70$ Guanine nucleotide binding protein mutations may have potential in differentiation of highest risk mucinous cysts from the more indolent. ${ }^{71}$ Neither are widely available. The 2018 ESGCTP guidelines advise that both can improve the detection of malignant change in mucinous cysts. There have been positive results using mass spectrometry of peptides such as mucin-5AC and mucin-2 to detect high risk mucinous cysts, though these findings require further validation. ${ }^{72}$

\section{Key points from this review}

- Pancreatic cystic lesions are common and accurate diagnosis can be difficult.

- The synthesis of clinical, radiological, biochemical and histopathological information needs to be prioritised over any single investigation.

- Correct differentiation between high-risk and low-risk $\mathrm{PCN}$ is important to guide appropriate allocation to surgery, surveillance or reassurance.

- Surgical options should be defined by location of lesion, patient factors and the malignant risk of the lesion. Mucinous cysts, in fit lesions, not meeting criteria for resection are surveilled.

- Further research is required to inform diagnostic, treatment and surveillance algorithms. 
Interest exists in minimally invasive therapies to halt malignant progression of mucinous cysts. Ablative therapies investigated include intracystic injection of ethanol and/or paclitaxel and radiofrequency ablation using a needle-based probe. These therapies remain investigative and should only be considered in non-operative candidates in trial settings. ${ }^{73}$

Contributors Research, tables and manuscript draft: MJK. Contribution to manuscript revisions and figures: MJK and BP.

Funding The authors have not declared a specific grant for this research from any funding agency in the public, commercial or not-for-profit sectors.

Competing interests None declared.

Patient consent for publication Not required.

Provenance and peer review Not commissioned; externally peer reviewed.

\section{REFERENCES}

1 de Jong K, Nio CY, Hermans JJ, et al. High prevalence of pancreatic cysts detected by screening magnetic resonance imaging examinations. Clin Gastroenterol Hepatol 2010;8:806-11.

2 Laffan TA, Horton KM, Klein AP, et al. Prevalence of unsuspected pancreatic cysts on MDCT. AJR Am J Roentgenol 2008;191:802-7.

3 Del Chiaro M, Besselink MG, Scholten L, et al. European evidence-based guidelines on pancreatic cystic neoplasms. Gut 2018;67:789-804.

4 Bosman FT, Carneiro F, Hruban RH, et al, 2010. WHO classification of tumours of the digestive system - NLM Catalog - NCBI. 4th ed. Lyon : IARC Press. Available: https:// www.ncbi.nlm.nih.gov/nlmcatalog/101553728 [Accessed 21 Jan 2018].

5 Procacci C, Biasiutti C, Carbognin G, et al. Characterization of cystic tumors of the pancreas: CT accuracy. J Comput Assist Tomogr 1999;23:906-12.

6 Pyke CM, van Heerden JA, Colby TV, et al. The spectrum of serous cystadenoma of the pancreas. Clinical, pathologic, and surgical aspects. Ann Surg 1992;215:132-9.

7 Al-Haddad M, Schmidt MC, Sandrasegaran K, et al. Diagnosis and treatment of cystic pancreatic tumors. Clin Gastroenterol Hepatol 2011;9:635-48.

8 Farrell JJ, Fernández-del Castillo C. Pancreatic cystic neoplasms: management and unanswered questions. Gastroenterology 2013;144:1303-15.

9 Le Borgne J, de Calan L, Partensky C. Cystadenomas and cystadenocarcinomas of the pancreas. Ann Surg 1999;230.

10 Hammel P, Voitot H, Vilgrain V, et al. Diagnostic value of Ca 72-4 and carcinoembryonic antigen determination in the fluid of pancreatic cystic lesions. Eur J Gastroenterol Hepatol 1998;10:345-8.

11 Belsley NA, Pitman MB, Lauwers GY, et al. Serous cystadenoma of the pancreas. Cancer 2008;114:102-10.

12 Galanis C, Zamani A, Cameron JL, et al. Resected serous cystic neoplasms of the pancreas: a review of 158 patients with recommendations for treatment. J Gastrointest Surg 2007;11:820-6.

13 Kimura W, Moriya T, Hirai I, et al. Multicenter study of serous cystic neoplasm of the Japan pancreas Society. Pancreas 2012;41:380-7.

14 Del Chiaro M, Verbeke C, Salvia R, et al. European experts consensus statement on cystic tumours of the pancreas. Dig Liver Dis 2013;45:703-11.

15 Katz MH, Mortenson MM, Wang H, et al. Diagnosis and management of cystic neoplasms of the pancreas: an evidencebased approach. J Am Coll Surg 2008;207:106-20.
16 Butte JM, Brennan MF, Gönen M, et al. Solid pseudopapillary tumors of the pancreas. Clinical features, surgical outcomes, and long-term survival in 45 consecutive patients from a single center. J Gastrointest Surg 2011;15:350-7.

17 Reddy S, Cameron JL, Scudiere J, et al. Surgical management of solid-pseudopapillary neoplasms of the pancreas (Franz or Hamoudi tumors): a large single-institutional series. J Am Coll Surg 2009;208:950-7.

18 Papavramidis T, Papavramidis S. Solid pseudopapillary tumors of the pancreas: review of 718 patients reported in English literature. J Am Coll Surg 2005;200:965-72.

19 Tanaka Y, Kato K, Notohara K, et al. Frequent beta-catenin mutation and cytoplasmic/nuclear accumulation in pancreatic solid-pseudopapillary neoplasm. Cancer Res 2001;61:8401-4.

$20 \mathrm{Al}$ M, Haddad - SMC, et al. Diagnosis and treatment of cystic pancreatic tumors. AJR Am J Roentgenol 2011;9:635-48.

21 Goh BK, Tan YM, Chung YF, et al. A review of mucinous cystic neoplasms of the pancreas defined by ovarian-type stroma: clinicopathological features of 344 patients. World J Surg 2006;30:2236-45.

22 Park JW, Jang JY, Kang MJ, et al. Mucinous cystic neoplasm of the pancreas: is surgical resection recommended for all surgically fit patients? Pancreatology 2014;14:131-6.

23 Scheiman JM, Hwang JH, Moayyedi P. American gastroenterological association technical review on the diagnosis and management of asymptomatic neoplastic pancreatic cysts. Gastroenterology 2015;148:824-48.

24 Farrell JJ, Prevalence FJJ. Prevalence, diagnosis and management of pancreatic cystic neoplasms: current status and future directions. Gut Liver 2015;9:571-89.

25 Yamao K, Yanagisawa A, Takahashi K, et al. Clinicopathological features and prognosis of mucinous cystic neoplasm with ovarian-type stroma: a multi-institutional study of the Japan pancreas Society. Pancreas 2011;40:67-71.

26 Tanaka M, Fernández-del Castillo C, Adsay V, et al. International consensus guidelines 2012 for the management of IPMN and McN of the pancreas. Pancreatology 2012;12:18397.

27 Brugge WR, Lauwers GY, Sahani D, et al. Cystic neoplasms of the pancreas. N Engl J Med Overseas Ed 2004;351:1218-26.

28 Grützmann R, Niedergethmann M, Pilarsky C, et al. Intraductal papillary mucinous tumors of the pancreas: biology, diagnosis, and treatment. Oncologist 2010;15:1294-309.

29 Fernández C, Castillo D, Volkan Adsay N. Mini-reviews and perspectives intraductal papillary mucinous neoplasms of the pancreas. YGAST;139:708-13.

30 Tanaka M, Fernández-del Castillo C, Kamisawa T, et al. Revisions of international consensus Fukuoka guidelines for the management of IPMN of the pancreas. Pancreatology 2017;17:738-53.

31 Basar O, Brugge WR. My treatment approach: pancreatic cysts. Mayo Clin Proc 2017;92:1519-31.

32 Stark A, Donahue TR, Reber HA, et al. Pancreatic cyst disease a review. J Am Med Assoc 2016;315:1882-93.

33 Robinson SM, Scott J, Oppong KW, et al. What to do for the incidental pancreatic cystic lesion? Surg Oncol 2014;23:11725.

34 Elta GH, Enestvedt BK, Sauer BG, et al. ACG clinical guideline: diagnosis and management of pancreatic cysts. Am J Gastroenterol 2018;113:464-79.

35 Swaroop Vege S, Ziring B, Jain R, et al. AGA section american gastroenterological association Institute guideline on the diagnosis and management of asymptomatic neoplastic pancreatic cysts and the clinical guidelines Committee. YGAST 2015;148:819-22.

36 Ghaneh P, Wong WL, Titman A, et al. PET-PANC: multi-centre prospective diagnostic accuracy and clinical value trial of FDG PET/CT in the diagnosis and management of suspected pancreatic cancer. JCO 2016;34(15_suppl). 
37 Kato K, Nihashi T, Ikeda M, et al. Limited efficacy of (18) F-FDG PET/CT for differentiation between metastasis-free pancreatic cancer and mass-forming pancreatitis. Clin $\mathrm{Nucl}$ Med 2013;38:417-21.

38 Strobel O, Büchler MW. Pancreatic cancer: FDG-PET is not useful in early pancreatic cancer diagnosis. Nat Rev Gastroenterol Hepatol 2013;10:203-5.

39 Zhang Y, Frampton AE, Martin JL, et al. 18F-fluorodeoxyglucose positron emission tomography in management of pancreatic cystic tumors. Nucl Med Biol 2012;39:982-5.

40 Sperti C, Pasquali C, Decet G, et al. F-18-fluorodeoxyglucose positron emission tomography in differentiating malignant from benign pancreatic cysts: a prospective study. $J$ Gastrointest Surg 2005;9:22-9.

41 Kim YC, Choi JY, Chung YE, Chul Kim Y, Eun Chung Y, et al. Comparison of MRI and endoscopic ultrasound in the characterization of pancreatic cystic lesions. AJR Am J Roentgenol 2010;195:947-52.

42 Leeds JS, Nayar MN, Dawwas M, et al. Comparison of endoscopic ultrasound and computed tomography in the assessment of pancreatic cyst size using pathology as the gold standard. Pancreatology 2013;13:263-6.

43 Adimoolam V, Sanchez MJ, Siddiqui UD, et al. Endoscopic ultrasound identifies synchronous pancreas cystic lesions not seen on initial cross-sectional imaging. Pancreas 2011;40:1070-2.

44 Brugge WR, Lewandrowski K, Lee-Lewandrowski E, et al. Diagnosis of pancreatic cystic neoplasms: a report of the cooperative pancreatic Cyst study. Gastroenterology 2004;126:1330-6.

45 van der Waaij LA, van Dullemen HM, Porte RJ. Cyst fluid analysis in the differential diagnosis of pancreatic cystic lesions: a pooled analysis. Gastrointestinal Endoscopy 2005;62:383-9.

46 Bick B, Enders F, Levy M, et al. The string sign for diagnosis of mucinous pancreatic cysts. Endoscopy 2015;47:626-31.

47 Walsh RM, Vogt DP, Henderson JM, et al. Management of suspected pancreatic cystic neoplasms based on cyst size. Surgery 2008;144:677-85. discussion 684-5.

48 Khashab MA, Kim K, Lennon AM, et al. Should we do EUS/ FNA on patients with pancreatic cysts? The incremental diagnostic yield of EUS over CT/MRI for prediction of cystic neoplasms. Pancreas 2013;42:717-21.

49 Nagayoshi Y, Aso T, Ohtsuka T, et al. Peroral pancreatoscopy using the SpyGlass system for the assessment of intraductal papillary mucinous neoplasm of the pancreas. J Hepatobiliary Pancreat Sci 2014;21:410-7.

50 Oppong KW, Dawwas MF, Charnley RM, et al. EUS and EUS-FNA diagnosis of suspected pancreatic cystic neoplasms: is the sum of the parts greater than the CEA? Pancreatology 2015;15:531-7.

51 Fritz S, Hackert T, Hinz U, et al. Role of serum carbohydrate antigen 19-9 and carcinoembryonic antigen in distinguishing between benign and invasive intraductal papillary mucinous neoplasm of the pancreas. Br J Surg 2011;98:104-10.

52 Jones NB, Hatzaras I, George N, et al. Clinical factors predictive of malignant and premalignant cystic neoplasms of the pancreas: a single institution experience. $H P B$ 2009;11:664-70.

53 Sperti C, Pasquali C, Guolo P, et al. Serum tumor markers and cyst fluid analysis are useful for the diagnosis of pancreatic cystic tumors. Cancer 1996;78:237-43.

54 Cheon YK, Cho YD, Jeon SR, et al. Pancreatic resection guided by preoperative intraductal ultrasonography for intraductal papillary mucinous neoplasm. Am J Gastroenterol 2010;105:1963-9.

55 Couvelard A, Sauvanet A, Kianmanesh R, et al. Frozen sectioning of the pancreatic cut surface during resection of intraductal papillary mucinous neoplasms of the pancreas is useful and reliable: a prospective evaluation. Ann Surg 2005;242:774-80.

56 Jang JY, Park T, Lee S, et al. Validation of international consensus guidelines for the resection of branch ducttype intraductal papillary mucinous neoplasms. Br J Surg 2014;101:686-92.

57 Roch AM, Ceppa EP, DeWitt JM, et al. International consensus guidelines parameters for the prediction of malignancy in intraductal papillary mucinous neoplasm are not properly weighted and are not cumulative. HPB 2014;16:929-35.

58 Aso T, Ohtsuka T, Matsunaga T, et al. "High-risk stigmata" of the 2012 international consensus guidelines correlate with the malignant grade of branch duct intraductal papillary mucinous neoplasms of the pancreas. Pancreas 2014;43:1239-43.

59 Goh BK, Thng CH, Tan DM, et al. Evaluation of the Sendai and 2012 international consensus guidelines based on crosssectional imaging findings performed for the initial triage of mucinous cystic lesions of the pancreas: a single institution experience with 114 surgically treated patients. Am J Surg 2014;208:202-9.

60 Malleo G, Marchegiani G, Borin A, et al. Observational study of the incidence of pancreatic and extrapancreatic malignancies during surveillance of patients with branch-duct intraductal papillary mucinous neoplasm. Ann Surg 2015;261:984-90.

61 Tanno S, Nakano Y, Sugiyama Y, et al. Incidence of synchronous and metachronous pancreatic carcinoma in 168 patients with branch duct intraductal papillary mucinous neoplasm. Pancreatology 2010;10:173-8.

62 Uehara H, Nakaizumi A, Ishikawa O, et al. Development of ductal carcinoma of the pancreas during follow-up of branch duct intraductal papillary mucinous neoplasm of the pancreas. Gut 2008;57:1561-5.

63 Schmidt CM, White PB, Waters JA, et al. Intraductal papillary mucinous neoplasms: predictors of malignant and invasive pathology. Ann Surg 2007;246:644-51.

64 Waters JA, Schmidt CM, Pinchot JW, et al. CT vs MRCP: Optimal classification of IPMN type and extent. In: Journal of Gastrointestinal Surgery. Springer-Verlag 2008:101-9.

65 Rodriguez JR, Salvia R, Crippa S, et al. Branch-duct intraductal papillary mucinous neoplasms: observations in 145 patients who underwent resection. Gastroenterology 2007;133:72-9.

66 Kang MJ, Jang JY, Lee KB, et al. Long-term prospective cohort study of patients undergoing pancreatectomy for intraductal papillary mucinous neoplasm of the pancreas: implications for postoperative surveillance. Ann Surg 2014;260:356-63.

$67 \mathrm{He}$ J, Cameron JL, Ahuja N, et al. Is it necessary to follow patients after resection of a benign pancreatic intraductal papillary mucinous neoplasm? J Am Coll Surg 2013;216:657-65.

68 Krishna SG, Brugge WR, Dewitt JM, et al. Needle-based confocal laser endomicroscopy for the diagnosis of pancreatic cystic lesions: an international external interobserver and intraobserver study (with videos). Gastrointest Endosc 2017;86:644-54.

69 Khalid A, Zahid M, Finkelstein SD, et al. Pancreatic cyst fluid DNA analysis in evaluating pancreatic cysts: a report of the panda study. Gastrointest Endosc 2009;69:1095-102.

70 Rockacy MJ, Zahid M, Mcgrath KM, et al. Liver, pancreas, and biliary tract association between KRAS mutation, detected in pancreatic cyst fluid, and long-term outcomes of patients. YJCGH 2013;11:425-9.

71 Singhi AD, Nikiforova MN, Fasanella KE, et al. Preoperative Gnas and KRAS testing in the diagnosis of pancreatic mucinous cysts. Clinical Cancer Research 2014;20:4381-9.

72 Jabbar KS, Arike L, Verbeke CS, et al. Highly accurate identification of cystic precursor lesions of pancreatic cancer through targeted mass spectrometry: a phase IIC diagnostic study. Journal of Clinical Oncology, 2018: 367-75.

73 Signoretti M, Valente R, Repici A, et al. Endoscopy-guided ablation of pancreatic lesions: technical possibilities and clinical outlook. WJGE 2017;9. 